\title{
Preparation of supercapacitors based on sludge utilization
}

\author{
Qingying Ye* \\ School of environment, North China Electric Power University, Baoding, China
}

\begin{abstract}
The acceleration of urbanization has made environmental problems increasingly prominent. With the continuous increase in the volume of urban sewage discharged annually, the problem of urban sludge pollution has gradually become an environmental problem in our country. At the same time, the expansion of grid-connected new energy has also continuously raised new challenges to low-cost and high-efficiency energy storage technology. This paper uses municipal sludge to prepare low-cost supercapacitors, that is, using municipal sludge as a raw material, through a series of physical and chemical processes such as drying, doping, impregnation, and activation, to prepare carbon-based porous electrode materials that can be used for supercapacitors. On the one hand, it solves the problem of sludge disposal. On the other hand, it greatly reduces the cost of supercapacitors and provides a new idea for preparing supercapacitors.
\end{abstract}

\section{Introduction}

With the acceleration of China's urbanization process, the discharge of urban sewage and sludge is increasing year by year. The sludge contains a lot of heavy metals, viruses, pathogens and other harmful substances. If improperly disposed of, it will cause serious secondary environmental pollution $[1,2]$. How to effectively treat urban sludge is a new issue that China faces after air and water pollution control. The main disposal methods of foreign sludge include sludge landfill, incineration and reclamation. European and American countries mainly use landfill and farmland. Japan's sludge from sea reclamation accounts for more than $70 \%$. At present, there are many domestic research methods for sludge treatment in China, including sanitary landfill, incineration, ocean dumping, anaerobic digestion, supercritical gasification, and hydrothermal treatment.

Supercapacitors are widely used in the new power industry due to their super fast charge and discharge capabilities and reliability. At present, China has not independently mastered the super capacitor technology, while foreign super capacitors are expensive and costly. There have been many studies on the preparation of activated carbon from sludge and the preparation of supercapacitors by using activated carbon, but there are few studies on the preparation of supercapacitors by directly using sludge. The reason is that the purity of the sludge carbon source is not high and ordinary activated carbon is difficult to meet the requirements of supercapacitors. Based on this background, this paper uses sludge as a raw material to prepare super electrode materials. The aim is to improve the purity of activated carbon from sludge through carbon source (straw) method, and use template method to realize porous carbon-based materials, and use modification methods to improve the specific energy density of the electrode of super electrode materials.

\section{Preparation process}

The entire preparation process roughly includes: after the collected municipal sludge is initially refined and purified, cellulose carbon sources such as straws are added for template design, and then the performance of the prepared electrode material is characterized. Under the diaphragm, the supercapacitor monolithic package is completed.

\subsection{Refining}

This part intends to use the activated carbonization method to prepare porous carbon-based materials. After drying, the municipal sewage sludge is crushed and screened (with straw blending), and then soaked in phosphoric acid solution for a certain period of time. The acidified sludge is subjected to shaking, pyrolysis, and carbonization treatment, and the phosphoric acid is recycled and reused. The processed sample is fully immersed in hot water and phosphoric acid is recovered at the same time. Finally, the carbon-based product is dried and milled, the whole process as shown in Figure 1.

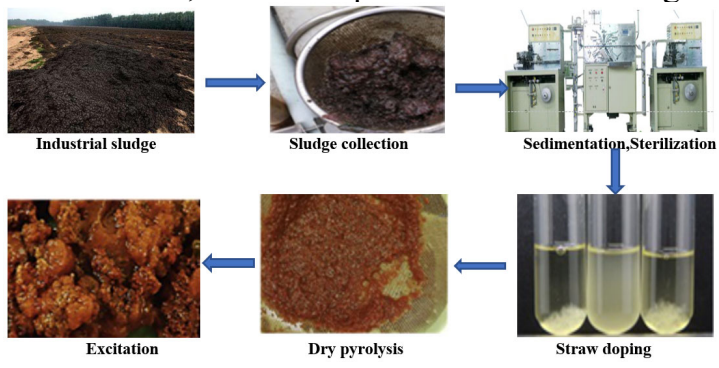

Fig. 1 Sludge scouring and purification process

\footnotetext{
* Corresponding author: 1265439827@qq.com
} 
Improved on the basis of traditional sludge purification activated carbon technology, taking into account the pseudocapacitance needs of supercapacitors, the treated sludge should maintain a certain amount of metalcontaining impurities (as composite or doping), which greatly reduces the traditional technical requirements.At the same time, it is also conducive to the subsequent doping modification process of electrode materials. The carbon-based material obtained through preliminary refining and purification has the characteristics of being fluffy, doped to a certain degree, and porous. However, it is not suitable for electrode materials of supercapacitors, and further chemical design and electrical performance improvement are required.

\subsection{Chemical design}

With the help of MS software, a variety of possible topologies can be designed. In this step, the research focuses from the macroscopic sludge to the microscopic single-molecule structure design. Using the template method, adding highly fibrous straw and waste paper for mixed treatment, so that the organic carbon molecules in the sludge grow along the highly fibrous carbon skeleton, and then gradually remove its hydrogen and oxygen during the subsequent activation carbonization process. Retaining carbon skeleton, its porous lattice structure and good thermal stability make the activated carbon at this stage have certain electrochemical properties.
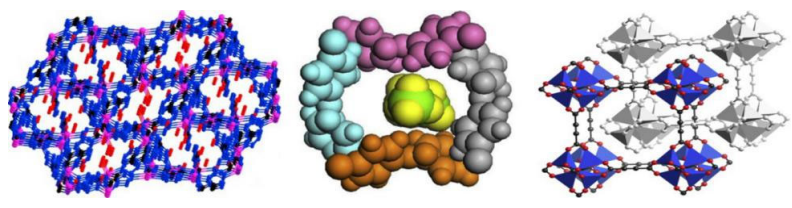

Fig 2. Micro-molecular structure design of sludge

Figure 3 shows the comparison of carbon-based materials before and after the molecular structure design of sludge under the electron microscope. The sludge sample designed by the template method is more dispersed and porous under the electron microscope, and has a larger specific surface area than before. Its ultra-microstructure enables it to have a complex ion channel unique to the super electrode material, which can hold more electrons. However, macroscopically, the battery performance is not yet known, and further measurement and characterization work is still needed.

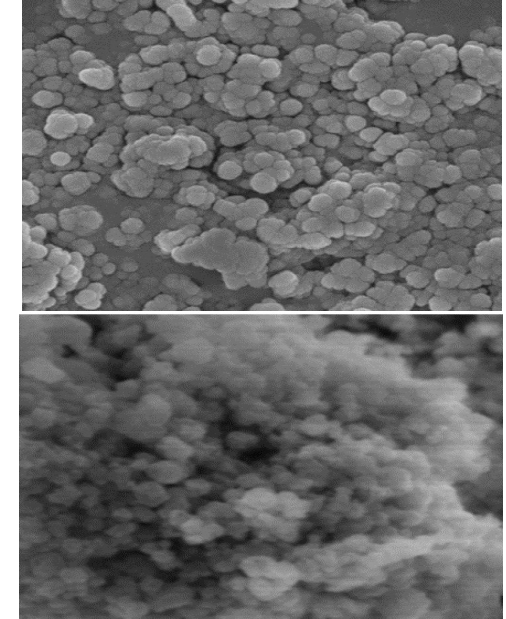

Figure 3. Comparison of carbon-based materials before and after sludge molecular structure design under electron microscope

\subsection{Research on the characteristics of supercapacitors}

At this stage, the development of high-energy-density electrodes must be realized. The carbon-based samples prepared above are combined with faraday pseudocapacitance materials, such as $\mathrm{MnO} 2, \mathrm{Ni}(\mathrm{OH}) 2$, etc[4].to prepare composite electrodes to obtain better specific capacitance characteristics. The electrode material after the composite pseudocapacitance material tends to have a higher porosity, and has a preliminary fibrous template, which indicates that the electrode material produced has preliminary requirements in terms of physical and chemical structure. However, further research on its electrochemical performance is still needed through electrochemical experiments.

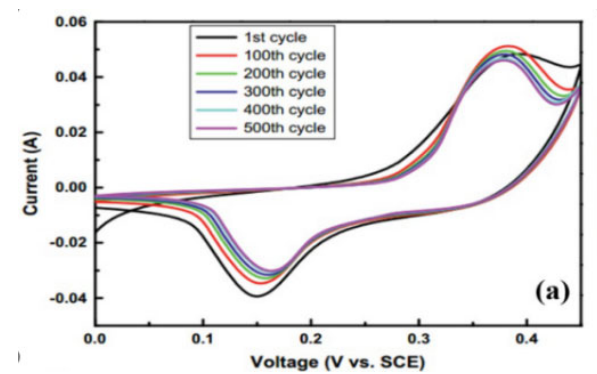

Fig 4. Cyclic Voltammetric Test of Capacitor Material

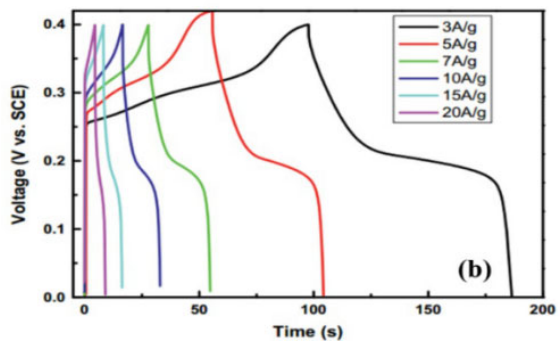

Fig.5 The relationship between the highest capacitance ratio and time under different electrolyte concentrations 
In order to verify the electrochemical characteristics of the fabricated electrode materials, electrochemical tests are required. From Figure 4, we can clearly see the uniform and symmetrical redox window, which proves the high reversibility of the charge and discharge of the material. Figure 5 shows that under different electrolyte adaptations, the highest specific capacitance can reach $265.6 \mathrm{~F} / \mathrm{g}$. This result can even be compared with some graphene parameters $(200 \sim 300 \mathrm{~F} / \mathrm{g})$ on the market.

At this time, the sludge electrode material we have developed has the characteristics of a super electrode. The next step is to focus on the adaptation of the electrolyte, so that the performance of the material can be thoroughly demonstrated and improved.

\subsection{Screening of electrolyte and diaphragm}

Study the coupling of organic electrolyte and sludge carbon-based materials and the selection of membrane materials, and carry out screening experiments on highseparation membrane materials with selective permeability to obtain membranes suitable for sludge carbon-based super electrodes. After hundreds of adaptation processes at this stage, we finally screened out the electrolyte and selective diaphragm that are supported by the sludge composite material, and its capacitance performance can be adequately guaranteed.

\subsection{Package molding}

After completing the synthesis of the electrode material, the capacitor needs to be encapsulated. Mix the electrode material, conductive additives and binder according to the proportions, and evenly coat them on a current collector such as foamed nickel. Use a tablet press to press the current collector sheet to a thickness of about $0.3 \mathrm{~mm}$, and wind it with a winding machine. In the vacuum glove box, the prepared electrode sheets are assembled into the super capacitor shell, electrolyte is injected, the diaphragm is added, and the aluminum shell is sealed with a sealing machine.

\subsection{Field test}

After completing the packaging of the capacitor monomer, in order to further study its energy storage performance and application prospects, with the cooperation of the Shanghai Electric Power Research Institute, the developed sludge supercapacitor was connected to the test bypass of its high-voltage main building for on-site assembly and test. The test results show that the operating temperature distribution is stable and the temperature rise is not obvious under normal operating conditions and long-term overload conditions, which is in line with the temperature rise of normal batteries. It shows that the capacitor meets the preliminary requirements of the power grid and has certain application value.

The power grid's requirements for the energy density of supercapacitors are not as high as those in the field of electric vehicles, so the use of sludge carbon-based materials to prepare supercapacitors can fully meet the performance requirements. At the same time, because the cost of supercapacitors is mainly concentrated in the synthesis and preparation of electrode materials, the use of sludge activated carbon can greatly save the expense of electrode materials. When the initial investment is the same, the supercapacitors made of this material will get better than traditional electrochemical supercapacitors. Large energy storage power and energy storage capacity, that is, higher cost performance.

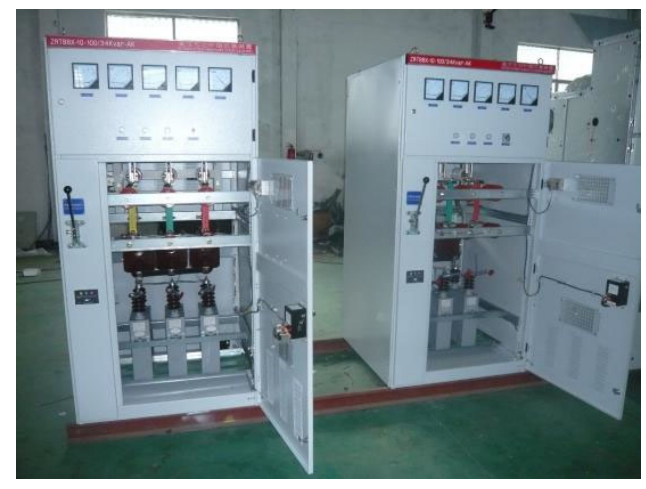

Figure 6. Field test and operation

\section{Conclusion}

In this paper, sludge is used as a raw material, through refining and purification, molecular design, electrochemical testing, electrolyte adaptation and encapsulation, and finally a supercapacitor material with higher specific energy density and higher cycle times is obtained. After on-site testing, it shows that it basically meets the requirements of the power grid.

After the super capacitor energy storage technology is applied to wind power and photovoltaic power generation, it can smooth the short-term power output fluctuations of the power station caused by unstable natural conditions such as wind and light. Due to the shortcomings of volatility, intermittentness, and unpredictability of renewable energy power generation, as well as traditional energy storage power sources used as wind power pitch power sources, power distribution network backup power sources have high maintenance costs and limited service life [5]. These all lead to long response time of physical energy storage and substandard power density of battery energy storage when compensating the pulse load of the grid. The output power density of the low-cost supercapacitor developed in this paper is as high as several $\mathrm{kW} / \mathrm{kg}$, and the cycle life can reach hundreds of thousands or even millions of times. It has reached the microgrid requirements in terms of cycle times, charging time, and safety, and has strong application value for the large-scale grid connection of new energy in the future.

\section{References}

1. Zhou Xuhong, Carbon nanotube-nickel hydroxide composite electrode electrochemical capacitor [J]. Electronic Components and Materials, 2015, 04: 16$18+21$.

2. Qian Jueshi, Xie Congbo, Xie Xiaoli, et al. Utilization status and research progress of urban 
sewage sludge as building materials. Journal of Building Materials, 2014, 17(5): 830 835.

3. Wang Dazhi, Preparation of nickel oxide/carbon nanotube supercapacitors from sludge $[\mathrm{J}]$. Chinese Journal of Inorganic Chemistry, 2013, 02:137-141.

4. Wang Xiaofeng, Electrochemical behavior of deposited nickel oxide and its supercapacitors[J]. Journal of Inorganic Materials, 2013, 02:331-336.

5. Chen Yingfang, Li Yuanyuan, Deng Meigen. The principle and application of supercapacitors[J]. Electronic Components and Materials, 2015, 04: 6-9. 